\title{
Effect of longitudinal density structure on a straight magnetic field modelling coronal arcade oscillations ${ }^{\star}$
}

\author{
G. R. Donnelly, A. J. Díaz ${ }^{\star \star}$, and B. Roberts
}

\begin{abstract}
Mathematical Institute, University of St Andrews, St Andrews, KY16 9SS, Scotland, UK
e-mail: [gavin; antonio; bernie] @mcs.st-and.ac.uk
\end{abstract}

Received 24 July 2006 / Accepted 5 June 2007

\section{ABSTRACT}

\begin{abstract}
Aims. Motivated by recent observations of oscillations in coronal arcades, we investigate analytically the influence of longitudinal structuring on the modes of oscillation of a straight coronal loop arcade. As a first step towards more complicated models, we use a simple structure to obtain analytical solutions.

Methods. A partial differential equation is derived for the total pressure perturbation of the fast modes in a zero beta plasma and it is solved analytically. We first recover the results for a homogeneous structure, and then study an equilibrium with an exponentially structured density profile, solving it in terms of Bessel functions of non-integer order and exponential argument, thus obtaining a dispersion relation. The properties of this dispersion relation are discussed and some limits studied, leading to analytical approximations to the eigenfrequencies.

Results. The introduction of longitudinal structuring results in a modification to the oscillatory frequencies of the modes of oscillation in such structures when compared with the uniform case. Regarding the oscillatory periods $P_{n}, n=1,2, \ldots$, the period ratios $P_{1} / 2 P_{2}$ and $P_{1} / 3 P_{3}$ are both shifted from unity. Other properties described in structured coronal loops are also found in an arcade: the occurrence of avoided crossings in the dispersion diagram and the displacement of the extrema towards the footpoints in the spatial structure of the eigenmodes.

Conclusions. We show analytically for simple arcade modes that the shift in the fundamental period proves to be small, but the ratio $P_{1} / 2 P_{2}$ depends strongly on the density structure. Moreover, transversal propagation also shifts the ratio $P_{1} / 2 P_{2}$ from unity, so it can be used in the coronal seismology of arcades in which transversal propagation is present. We use the currently available observational data to illustrate this application.
\end{abstract}

Key words. Sun: oscillations - Sun: magnetic fields - Sun: corona

\section{Introduction}

There is a wide variety of structures in the solar corona, with the basic building block for these structure being magnetic flux tubes. Many of these structures display oscillatory behaviour (see Aschwanden 2004 and Nakariakov \& Verwichte 2005, for detailed overviews). Observations of oscillations have been interpreted using theoretical models (see for example Edwin \& Roberts 1983; Roberts et al. 1984; Nakariakov \& Ofman 2001).

Observations reveal that flux tubes often occur in groups, forming structures such as coronal arcades. These arcades are often formed in the aftermath of a flare or a flux rope eruption. In its simplest form, a coronal arcade consists of a set of closely packed loops of equal length which run parallel to each other connecting the two elongated parallel footpoints or ribbons which anchor the arcade to the photosphere (see Fig. 1 for a sketch of the structure). In reality, a coronal arcade may be made up of loops which do not lie parallel to each other, being inhomogeneous and possessing differing plasma properties from each other. The arcade may be comprised of loops differing in length, thickness and radius of curvature.

As with the majority of coronal structures, coronal arcades have been observed to support MHD oscillations (Aschwanden et al. 2002). Of particular interest here are the observations of

\footnotetext{
* Appendix A is only available in electronic form at http://www . aanda.org

$\star \star$ Current address: Departament de Física, Universitat de les Illes Balears, E07122, Palma, Spain.
}

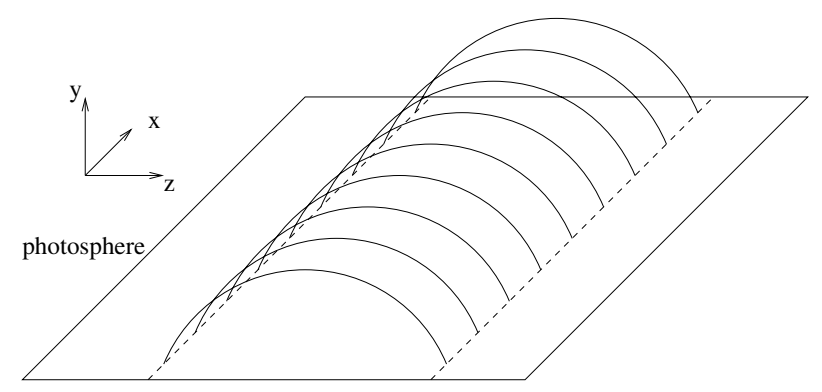

Fig. 1. Sketch of magnetic field lines forming a coronal arcade which connects two ribbons in the photosphere. The arcade is assumed to be invariant in the $x$-direction.

oscillations of a post-flare loop arcade detected in TRACE data on the 15th of April 2001 (Verwichte et al. 2004). An analysis of the oscillatory properties of nine loops within the arcade were made, finding that the loops lie in the height range $65-76 \mathrm{Mm}$ with periods of oscillation in the loops found to lie in the range 200-450 s (determined consistently using a wavelet analysis and also a curve fitting method). The damping times were in the range $800-1800 \mathrm{~s}$. Examination of the periods and loop displacements has shown that not only the fundamental mode but also its first harmonic has been observed in two of the loops within the arcade. The ratio of the periods of the fundamental and its first harmonic have been discussed and suggested as a useful tool in coronal seismology (Andries et al. 2005b; 
McEwan et al. 2006; Goossens et al. 2006). The oscillation periods and the observed transverse nature of the loop displacement is consistent with that of the kink mode.

The role of curvature on coronal arcades has been investigated in recent years. Oliver et al. (1993) studied analytically the oscillatory modes of a stratified potential arcade. The modes of a curved arcade have been further explored both analytically and numerically, focusing on the differences when compared with straight models (Smith et al. 1997; Brady \& Arber 2005; Selwa et al. 2005; Verwichte et al. 2006a,b; Díaz et al. 2006b; Díaz 2006). These works show that leakage is an important effect resulting from curvature. Other interesting effects are also present, such as the appearance of new families of modes vertically or horizontally polarised, or the presence of avoided crossings with the modes of the cavity below the arcade (Díaz et al. 2006b).

The effect of longitudinal structure along the magnetic field on straight loops has also drawn recent interest (Díaz et al. 2004; Andries et al. 2005a,b; Díaz et al. 2006a; McEwan et al. 2006; Dymova \& Ruderman 2006; Erdélyi \& Verth 2007). It has been shown that structure does not affect significantly the frequency of the fundamental mode, but higher harmonics are modified more substantially. Propagating modes have not been considered in the context of these works.

Our aim here is to discuss analytically the effect of longitudinal structure on the vertically polarised mode, which is a transverse oscillation of the arcade. We carry out this study using a 2D Cartesian geometry in a straightened arcade and examine the influence of longitudinal structuring on these modes of oscillation. Finally, we compare our results with the periods reported by Verwichte et al. (2004).

\section{Equilibrium and basic equations}

We are interested in the role of density structuring along the magnetic field lines of a coronal arcade. The complex geometry of an arcade has lead us to explore the role of density structuring in a straight magnetic field. We consider a uniform equilibrium magnetic field $\boldsymbol{B}_{0}=B_{0} \boldsymbol{e}_{z}$ which lies along the $z$ axis of a Cartesian coordinate system. The equilibrium plasma density $\rho_{0}(z)$ is permitted to vary along the field, reflecting the role of stratification in an arcade. To reflect the finite length of an arcade's magnetic field lines anchored in the photosphere, we suppose that the field lines are line-tied at $z= \pm L$ (so the overall length of the arcade is $2 L$ ). The arcade is considered to be inifinite in the direction perpendicular to the magnetic field, with no variation in the horizontal $y$-direction. The direct effect of gravity is, however, ignored. We study the modes of oscillation of this Cartesian equilibrium, allowing propagation across the magnetic field. The resulting set of linear equations can be written as a set of four coupled partial differential equations for the primary variables: total pressure perturbation $P_{T}\left(=P+B_{0} B_{z} / \mu\right)$, the two components of the perpendicular velocity and the longitudinal velocity $v_{z}$. We define $v_{A}(z)=B_{0} / \sqrt{\mu \rho_{0}(z)}$ and $c_{0}(z)=$ $\sqrt{\gamma P_{0} / \rho_{0}(z)}$, the Alfvén and sound speeds; $P$ denotes the perturbation in the plasma pressure and $B_{z}$ is the component of the perturbed magnetic field in the $z$-direction. Equations of this form have been discussed in Roberts (1991), Díaz (2004) and Díaz et al. (2006a).

The corona is magnetically dominated with a low plasma $\beta=\left(2 c_{0}^{2}\right) /\left(\gamma v_{A}^{2}\right)$. The limit of $\beta=0$ eliminates acoustic effects (including the slow mode), but allows a clearer investigation into the behaviour of the fast waves, unhindered by the complexities introduced by acoustic effects. In this geometry the Alfvén mode is decoupled, allowing us to carry out a study of the fast mode. It is important to note that we make this approximation in the corona but also in the chromosphere/photospheric level, where it is less appropriate and gravitational effects are significant. We consider an exponential longitudinal density profile, representing the rapid change from the dense photosphere to the rarefied corona, while the density is uniform in the transverse direction. We assume symmetry of the equilibrium about the $x$ axis and invariance of the perturbation in the $y$-direction, so $\frac{\partial}{\partial y}=0$.

With $\beta=0$, the governing equations may be reduced to the wave equation for $P_{T}$ (Díaz et al. 2002; Díaz 2004; Díaz et al. 2006a)

$$
\left[\frac{\partial^{2}}{\partial t^{2}}-v_{A}^{2}(z) \nabla^{2}\right] P_{T}=0 .
$$

There is no perturbation in the velocity along the field lines $\left(v_{z}=\right.$ 0 ) and the motion $\boldsymbol{v}=v_{x} \boldsymbol{e}_{x}$ satisfies

$\rho_{0}(z)\left[\frac{\partial^{2}}{\partial t^{2}}-v_{A}^{2}(z) \frac{\partial^{2}}{\partial z^{2}}\right] v_{x}=-\frac{\partial^{2} P_{T}}{\partial x \partial t}$

Motions of the arcade footpoints as a result of oscillations within the corona are minimal (Hood 1986): the low coronal density (in comparison with the photosphere) results in coronal oscillations carrying relatively little momentum, leaving the photosphere unperturbed. This leads us to impose the line-tying boundary condition $v_{x}(z= \pm L)=0$. Thus, there are no transverse oscillations at the footpoints $(z= \pm L)$ of the arcade.

We study solutions to Eqs. (1) and (2) which are oscillatory in time, so a Fourier form is taken for the time component. The modes we are interested in are freely propagating perpendicular to the magnetic field and so a Fourier analysis in the $x$-direction may be made. This leads to $P_{T}$ having the form

$P_{T}(x, z, t)=h(z) \exp [\mathrm{i}(\omega t+k x)]$

with frequency $\omega$ and wavenumber $k$ in the $x$-direction. The amplitude $h(z)$ depends upon distance $z$ along the field, in which the arcade is structured. Substituting (3) into Eq. (1) results in the ordinary differential equation

$$
\frac{\mathrm{d}^{2} h}{\mathrm{~d} z^{2}}+\left(\frac{\omega^{2}}{v_{A}^{2}(z)}-k^{2}\right) h=0 .
$$

Using a Fourier decomposition for the transverse velocity similar to Eq. (3), $v_{x}$ and $P_{T}$ are related from Eq. (2) by

$$
v_{x}=\frac{-c}{\rho_{0}(z) v_{A}^{2}(z)} P_{T},
$$

where $c(=\omega / k)$ is the phase speed along the arcade. Note that $\rho_{0}(z) v_{A}^{2}(z)=B_{0}^{2} / \mu$ is a constant.

Another essential point about the interfaces in the equilibrium models discussed in this paper is that they are perpendicular to the equilibrium magnetic field. The boundary conditions at such an interface are (Goedbloed 1983; Díaz 2004):

$[\boldsymbol{v}]=[\boldsymbol{B}]=\left[P_{T}\right]=0$,

where $[\mathbf{a}]=\mathbf{a}_{2}-\mathbf{a}_{1}$, and the subscripts 1 and 2 represent the quantity on either side of the interface. Equation (6) implies that all velocity and magnetic field components as well as the total pressure must be continuous at such an interface. 


\section{Results}

We discuss the solutions of Eqs. (1) and (2) for different equilibrium density profiles. Firstly, we consider a profile with constant density along the arcade giving simple mathematical expressions which may be used as a comparison with more complicated models. We study an exponentially structured environment, which models better the equilibrium of solar arcades and leads to more complex mathematical dispersion relations which are solved numerically. Finally, we combine both models to study an arcade with a an exponentially decreasing equilibrium density profile near the footpoints and a region with constant density in the summit, representing the arcade top.

\subsection{Homogeneous medium}

We begin our discussion by considering the case of a homogeneous medium. In a uniform medium the Alfvén speed $v_{A}(z)=$ $v_{A 0}$ is a constant, so there is no variation in density or Alfvén speed in either the parallel or perpendicular directions with respect to the magnetic field. Hence we may write Eq. (4) as

$\frac{\mathrm{d}^{2} h}{\mathrm{~d} z^{2}}+M_{0}^{2} h=0$,

where $M_{0}^{2}=\frac{\omega^{2}}{v_{A 0}^{2}}-k^{2}$ is a constant.

In order to satisfy the line-tying boundary conditions $h(z=$ $\pm L)=0$ the solutions to Eq. (7) must be oscillatory in nature; hence $M_{0}^{2}>0$. This implies a cutoff value for the frequency, since $\omega>v_{A 0} k$ (or in terms of the phase speed $c>v_{A 0}$ ). If we separate solutions for $h(z)$ into modes which are even or odd in the $z$-direction, then

$h(z)= \begin{cases}A_{\mathrm{s}} \sin \left(M_{0} z\right), & \text { odd modes } \\ A_{\mathrm{c}} \cos \left(M_{0} z\right), & \text { even modes }\end{cases}$

with $A_{\mathrm{s}}$ and $A_{\mathrm{c}}$ being arbitrary constants. Application of the linetying conditions at $z=L$ implies that

$\sin \left(M_{0} L\right)=0, \quad$ odd modes,

$\cos \left(M_{0} L\right)=0, \quad$ even modes.

Hence, taken together we obtain

$M_{0} L=n \frac{\pi}{2}, \quad n=1,2 \ldots$

Thus, for these modes we obtain frequencies $\omega=\omega_{n}$ described by the equation

$\frac{\omega_{n}^{2}}{v_{A 0}^{2}}-k^{2}=\left(\frac{n \pi}{2 L}\right)^{2}, \quad n=1,2, \ldots$,

so the phase speed $c=c_{n}$ satisfies

$\left(\frac{c_{n}}{v_{A 0}}\right)^{2}=\left(\frac{n \pi}{2 k L}\right)^{2}+1, \quad n=1,2, \ldots$

Equation (12) encompasses both even and odd modes, with the even modes corresponding to odd values of the integer $n$ and odd modes corresponding to even values of $n$. From dispersion relation in Eq. (12) we see that both even and odd modes tend to lower limit $c / v_{A 0}=1$ in the short wavelength limit $k L \rightarrow \infty$. Figure 2a illustrates the behaviour of (12).

Very recently the importance of the ratio $P_{1} / 2 P_{2}$ of the periods of the fundamental mode $\left(P_{1}\right)$ and its first harmonic $\left(P_{2}\right)$ has

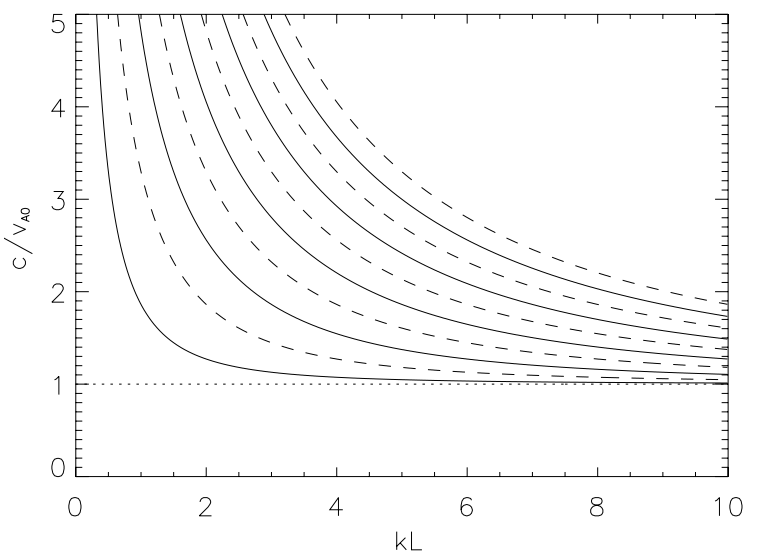

(a)

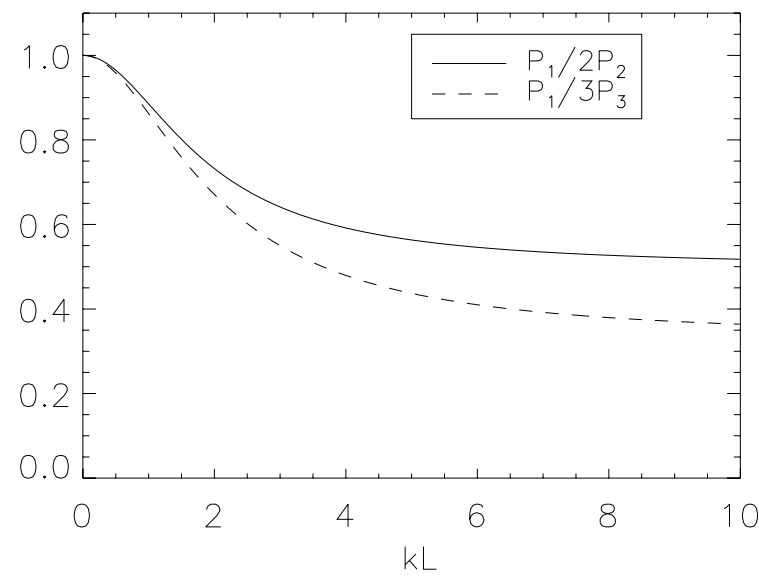

(b)

Fig. 2. a) Dispersion diagram plotting the dimensionless phase speed $c / v_{A 0}$ against the dimensionless perpendicular wavenumber $k L$ for a homogeneous arcade. The even and odd modes are plotted as solid and dashed curves, respectively. The dotted horizontal line $c / v_{A 0}=1$ marks the cutoff curve. b) The ratio of the fundamental period $P_{1}$ and its first harmonic $P_{2}$, namely $P_{1} / 2 P_{2}$ (solid line), and the ratio of the fundamental period $P_{1}$ and its second harmonic $P_{3}$, namely $P_{1} / 3 P_{3}$ (dashed line), as a function of $k L$.

been discussed in the context of coronal seismology (see Andries et al. 2005b; Goossens et al. 2006; McEwan et al. 2006). In this simple case we can obtain an explicit formula for the period ratio $P_{n} / P_{m}$ :

$\left(\frac{P_{n}}{P_{m}}\right)^{2}=\left(\frac{\omega_{m}}{\omega_{n}}\right)^{2}=\frac{(m \pi)^{2}+4(k L)^{2}}{(n \pi)^{2}+4(k L)^{2}}$,

for integers $n$ and $m$. In particular, setting $n=1$ and $m=2$ gives

$\frac{P_{1}}{2 P_{2}}=\left[\frac{\pi^{2}+(k L)^{2}}{\pi^{2}+(2 k L)^{2}}\right]^{1 / 2}$.

In the limit $k L \rightarrow 0, P_{1} / 2 P_{2} \rightarrow 1$ and as $k L \rightarrow \infty, P_{1} / P_{2} \rightarrow 1 / 2$. These trends can be observed in Fig. $2 \mathrm{~b}$, which plots $P_{1} / 2 P_{2}$ as a function of $k L$.

\subsection{Exponential profile}

Next we examine the influence of an exponentially structured environment. In the corona, this structuring could arise as a result of gravity or temperature inhomogeneities; here we incorporate the structuring of the environment in the equilibrium model but 
neglect the dynamical effects which produces the structuring in the wave analysis. We consider an Alfvén profile of the form

$v_{A}(z)=v_{A 0} \exp (-\alpha|z| / L)$,

which gives $v_{A}(z)$ decaying exponentially (for $\alpha>0$ ) from the loop apex $(z=0)$ towards the photospheric footpoint $(z= \pm L)$. Such a profile has a sharp peak at the loop apex, were the boundary conditions in Eq. (6) are valid. However, we restrict our analysis to the domain $0 \leq z \leq L$ on grounds of symmetry.

\subsubsection{Dispersion relations}

Using the Alfvén profile (15), Eq. (4) takes the form

$\frac{\mathrm{d}^{2} h}{\mathrm{~d} z^{2}}+\left(\frac{\omega^{2}}{v_{A 0}^{2}} \exp (2 \alpha z / L)-k^{2}\right) h=0$,

for $z \geq 0$. Equation (16) has general solution (Abramowitz \& Stegun 1964)

$$
\begin{aligned}
h(z)=A_{1} & J_{v}\left(\frac{c k L}{\alpha v_{A} 0} \exp (\alpha z / L)\right) \\
& +B_{1} Y_{v}\left(\frac{c k L}{\alpha v_{A 0}} \exp (\alpha z / L)\right), \quad z \geq 0 .
\end{aligned}
$$

where $J$ and $Y$ denote Bessel functions of order $v=k L / \alpha$ of the first kind and $A_{1}$ and $B_{1}$ are arbitrary constants The Bessel functions in Eq. (17) are generally of non-integer order $v$, depending on $k L$ and $\alpha$. We also note that in the region $[-L, L]$ neither Bessel function is singular, since their arguments are exponential functions of $z$ (hence $Y_{v}(0)$ is never attained); therefore, both Bessel functions of the first kind are retained in the solution for $h(z)$.

Due to the symmetry of the equilibrium it is again possible to separate solutions into even and odd modes, depending on the disturbances they give rise to about $z=0$. The boundary conditions for the complete problem are

$$
\begin{aligned}
& h(z= \pm L)=0, \\
& \lim _{z \rightarrow 0^{-}} h(z)=\lim _{z \rightarrow 0^{+}} h(z), \\
& \lim _{z \rightarrow 0^{-}} \frac{\mathrm{d} h}{\mathrm{~d} z}=\lim _{z \rightarrow 0^{+}} \frac{\mathrm{d} h}{\mathrm{~d} z} .
\end{aligned}
$$

The first condition arises because of the line-tying; the second condition follows from the continuity of $P_{T}$ (or $v_{x}$ ) across the apex $z=0$ (Eq. (6)). The third condition is a consequence of the continuity of the perturbed magnetic field from Eq. (6), which imply that $\frac{\partial v_{x}}{\partial z}$ (and therefore $\frac{\mathrm{d} h}{\mathrm{~d} z}$ ) are continuous across $z=0$. Once we have separated solutions into even and odd modes, the boundary conditions are reduced in each case since some are identically satisfied, with

$\lim _{z \rightarrow 0} h(z)=0$

for odd modes, and

$\lim _{z \rightarrow 0} \frac{\mathrm{d} h}{\mathrm{~d} z}=0$

for even modes. The conditions in Eqs. (19) and (20) are in addition to the line-tying condition $h=0$ at $z \pm L$.

Application of these boundary condition yield the following dispersion relations:

$$
\begin{aligned}
J_{v}\left(\frac{c k L}{\alpha v_{A 0}} \exp (\alpha)\right) Y_{v}\left(\frac{c k L}{\alpha v_{A 0}}\right) \\
-J_{v}\left(\frac{c k L}{\alpha v_{A 0}}\right) Y_{v}\left(\frac{c k L}{\alpha v_{A 0}} \exp (\alpha)\right)=0
\end{aligned}
$$

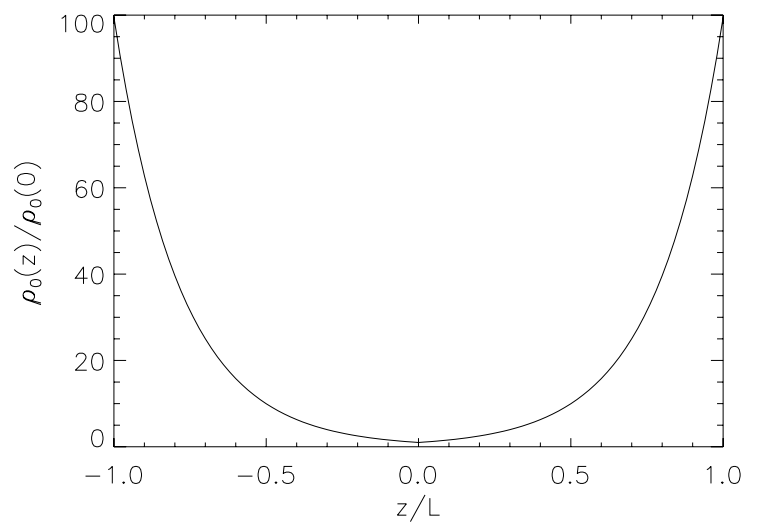

Fig. 3. The dimensionless density profile $\rho_{0}(z) / \rho_{0}(0)$ as a function of $z / L$ for an exponentially stratified arcade with the base density 100 times larger that the apex density, $\rho_{0}(L) / \rho_{0}(0)=100$.

for the odd modes, and

$$
\begin{aligned}
J_{v}\left(\frac{c k L}{\alpha v_{A 0}} \exp (\alpha)\right) Y_{v}^{\prime}\left(\frac{c k L}{\alpha v_{A 0}}\right) \\
-J_{v}^{\prime}\left(\frac{c k L}{\alpha v_{A 0}}\right) Y_{v}\left(\frac{c k L}{\alpha v_{A 0}} \exp (\alpha)\right)=0
\end{aligned}
$$

for the even modes. Here the dash denotes the derivative of the Bessel function. These are transcendental equations for the phase speed $c=\omega / k$ perpendicular to the equilibrium magnetic field (in the $x$-direction) and describe the modes of oscillation exhibited by a coronal arcade stationary in the longitudinal direction but freely propagating in the transverse component, with an exponential density profile. Dispersion relations Eqs. (21) and (22) are similar in form and are closely related to those found by McEwan et al. (2006) and Díaz \& Roberts (2006). In these papers, dispersion relations involving Bessel functions of exponential arguments are derived for standing slow modes in a coronal loop with and equilibrium density profile similar to the exponential one discussed in our paper and by using a thin tube approximation or a stretching coordinate. However, in a coronal arcade longitudinal propagation is an important feature not present in these studies for the slow mode of coronal loops, so in McEwan et al. (2006); Díaz \& Roberts (2006) the order of the Bessel functions is a mere integer, while in Eqs. (21) and (22) the order of the Bessel functions $v$ depends of the longitudinal wavenumber $k$ and, therefore, is not necessary an integer.

\subsubsection{Numerical solutions to the dispersion relations}

We now present plots of the dispersion curves and eigenfunctions $P_{T}$, choosing the density profile shown in Fig. 3 . We choose a density enhancement,

$\rho_{0}(L) / \rho_{0}(0)=\exp 2 \alpha$,

by a factor of 100 between the photospheric plasma equilibrium density at the footpoints $(z= \pm L)$ and the loop apex $(z=0)$, so $\alpha=2.3$.

Figure 4a displays the dispersion diagram for the fundamental mode and its first nine harmonics, showing both even (solid) and odd (dashed) modes. There is no upper cutoff frequency, since the Alfvén profile is invariant in the $x$-direction; hence, all modes propagate for all $k L$ with no mode propagating with finite phase speed in the short wavelength limit. Notice that this behaviour is different from the results in Edwin \& Roberts (1983) 


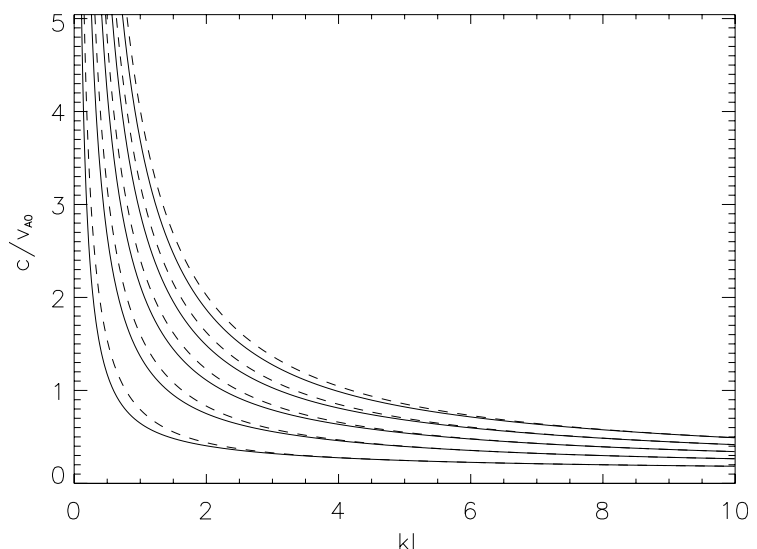

(a)

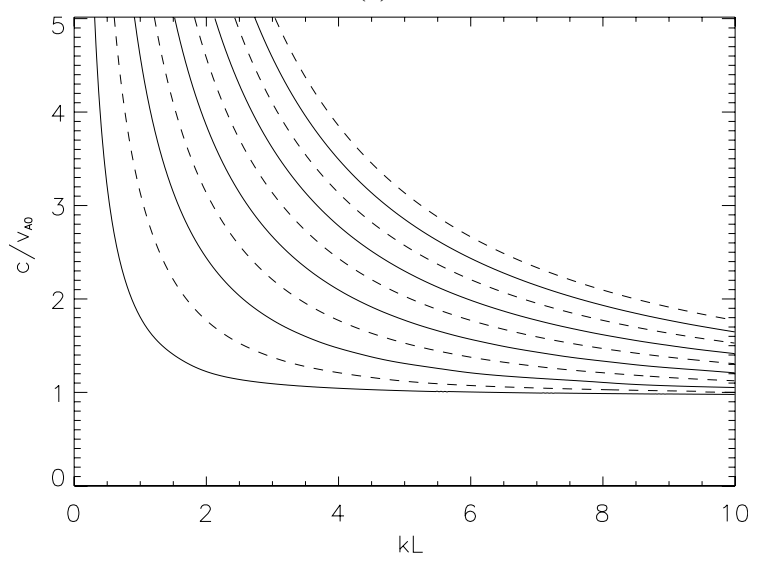

(b)

Fig. 4. Dispersion diagrams plotting the dimensionless phase speed $c / v_{A 0}$ against $k L$ for an exponentially stratified arcade, with a) $\rho_{0}(L) / \rho_{0}(0)=100$ and $\left.\mathbf{b}\right) \rho_{0}(L) / \rho_{0}(0)=1.2$. The even modes are shown as solid curves, and the odd modes as dashed curves. Only the 10 lowest values of $c / v_{A 0}$ have been represented.

for a coronal loop, where the fundamental mode is propagating in the short wavelength limit while the other modes have a cutoff; but here propagation is across the arcade (in the $y$-direction), while in Edwin \& Roberts (1983) propagation is along the loop (the $z$-direction). The phase speeds of consecutive even and odd modes rapidly converge when $k L$ is increased. As in the homogeneous case there is a lower limit to which the phase speed tends to as $k L \rightarrow \infty$. It can be argued from Eq. (16) that the lower limit lies in the range $[\exp (-\alpha), 1]$, since the solution to Eq. (16) must have a region where it exhibits oscillatory behaviour in $[0, L]$, in order to satisfy the line-tying boundary condition. This oscillatory behaviour can only occur when the quantity in parenthesis in Eq. (16) is positive, so

$\frac{\omega^{2}}{v_{A 0}^{2}} \exp (2 \alpha z / L)-k^{2}>0$,

which leads to

$\frac{c}{v_{A 0}}>\exp (-\alpha z / L)$

Hence the ends of this interval $z=0$ and $z=L$ give the extreme values that the cutoff could possibly take. Note that we recover the homogeneous limit by allowing $\alpha \rightarrow 0$. It will be demonstrated later that $c / v_{A 0} \rightarrow \exp (-\alpha)$ as $k L \rightarrow \infty$.

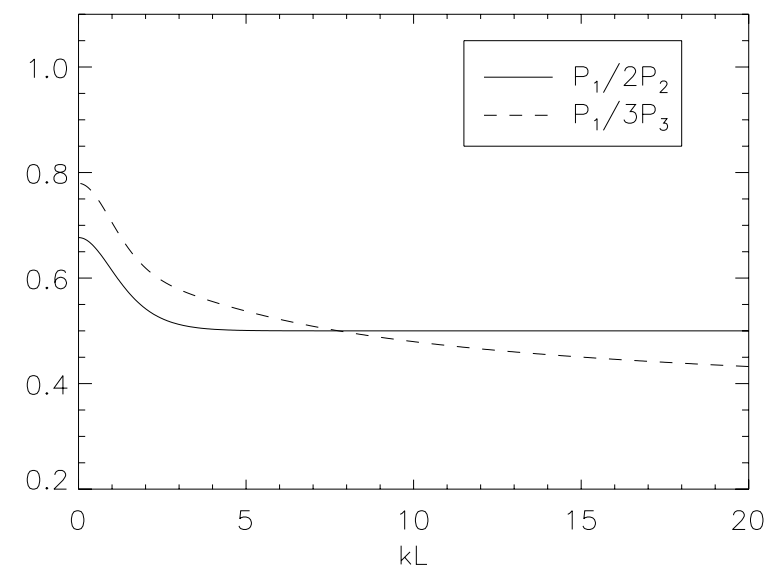

Fig. 5. The ratio $P_{1} / 2 P_{2}$ of the fundamental even mode of period $P_{1}$ and the odd mode harmonic of period $P_{2}$ (solid line), and the ratio $P_{1} / 3 P_{3}$ of the fundamental even mode of period $P_{1}$ and the even mode harmonic of period $P_{3}$ (dashed line), plotted against $k L$ for an exponentially stratified arcade with $\rho_{0}(L) / \rho_{0}(0)=100(\alpha=2.3)$.

An important feature recently discussed by Andries et al. (2005b) and McEwan et al. (2006) is that the ratio of the periods of the fundamental and its first harmonic differ from 2 . This trend is evident in Fig. 5, which displays $P_{1} / 2 P_{2}$ as a function of $k L$. Note that $P_{1} / 2 P_{2}$ involves a comparison between the first even mode (the fundamental mode) and the first odd mode (the first harmonic of the system). We see that for $P_{1} / 2 P_{2}<1$ for all $k L$. Here, with $\alpha=2.3$, we find that $P_{1} / 2 P_{2} \leq 0.68$ with the value of $P_{1} / 2 P_{2}=0.68$ attained in the long wavelength limit $(k L \rightarrow 0) . P_{1} / 2 P_{2}$ is a decreasing function of $k L$, tending to $1 / 2$ in the short wavelength limit $(k L \rightarrow \infty)$. The limit $\alpha \rightarrow 0$ recovers the results for a uniform medium (Fig. 2b), as seen in Fig. $4 \mathrm{a}$, and $P_{1} / 2 P_{2}$ falls off towards the limiting value $1 / 2$ as $k L$ is increased.

The ratio between the fundamental mode $(n=1)$ and its second harmonic $(n=3)$, which is also an even mode, $P_{1} / 3 P_{3}$, has also been included in Fig. 5. We can check that $P_{1} / 3 P_{3}<1$, that it decreases monotonically, and that in the short wavelength limit $(k L \rightarrow \infty)$ it tends to $1 / 3$, similarly to the behaviour of $P_{1} / 3 P_{3}$ for a homogeneous arcade (see Fig. 2b).

Figure 6 a shows plots of the total pressure perturbation (in units of $\left.\rho_{0} v_{A 0}^{2}\right), P_{T} /\left(\rho_{0} v_{A 0}^{2}\right)$, against $z / L$ for the fundamental mode and the first and second harmonics of the even modes; here $k L=2.0$. The fundamental mode does not display the expected behaviour as it has three extrema rather than a single extremum which would occur in the uniform arcade case. Comparing the solid and dotted curves in Fig. 6a we see that the total pressure perturbation has a minimum at the loop apex $(z=0)$; this appears to be a characteristic of modes which have phase speed less than the maximum Alfvén speed, $c<v_{A 0}$. This trend is followed by all of the modes plotted in Fig. 6b, which are equivalent to those of Fig. $6 \mathrm{a}$ but for $k L=8$. Also, from the fundamental mode plotted in Fig. 6a we see growing numbers of rapid oscillations near the footpoints, for both $k L=2$ and $k L=8$; the amplitude of these oscillations falls from the apex to the footpoints, so the oscillation power has been mainly restricted to the denser region of the loop (near the footpoints situated at $z= \pm L$ ) and the effect is seen more strongly for the case $k L=8$ shown in Fig. $6 \mathrm{~b}$, with the oscillation causing almost no perturbation to the loop apex. This is in accordance with the results of similar density profiles for the slow mode, which also show these oscillations as the 


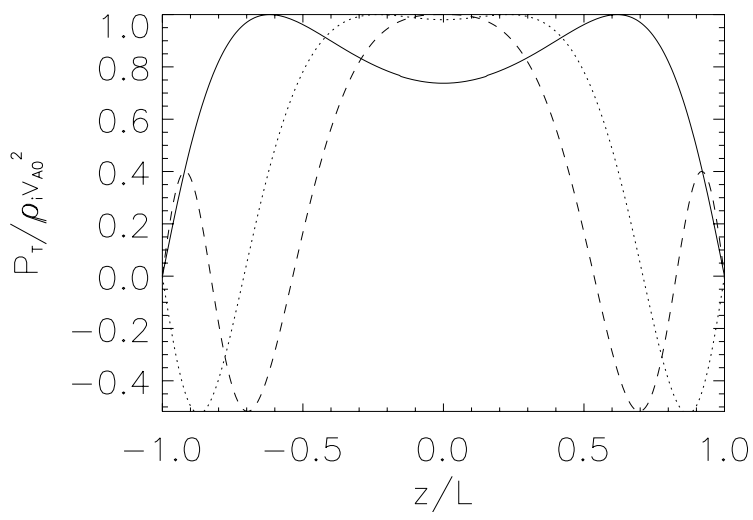

(a)

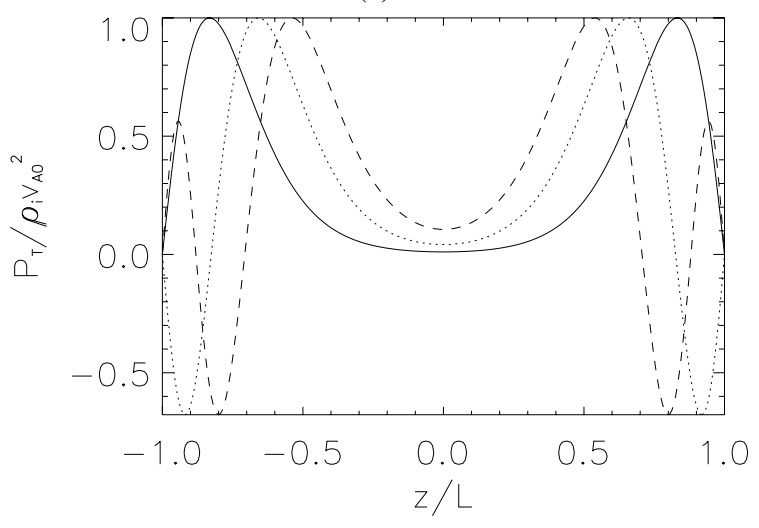

(b)

Fig. 6. Plots of the dimensionless total pressure perturbation $P_{T} /\left(\rho_{0} v_{A 0}^{2}\right)$ against $z / L$ for the fundamental (solid curve), first even harmonic (dotted curve) and second even harmonic (dashed curve), for a) $k L=2$ and b) $k L=8$. An exponential density profile is used with density enhanced by a factor of 100 between the loop apex and the footpoints.

density contrast between footpoints and apex is increased (Díaz \& Roberts 2006).

\subsubsection{Analytical approximations for the dispersion relations}

We now examine the dispersion relations in Eqs. (21) and (22) analytically. Firstly, using the expansions in Eq. (9.5.28) (p. 374) of Abramowitz \& Stegun (1964) for the zeros of the expression

$J_{v}(a) Y_{v}(\lambda a)-J_{v}(\lambda a) Y_{v}(a)$

we can derive an approximate dispersion relation for the odd harmonics multiplying Eq. (21) by -1 and comparing it to Eq. (26):

$$
\begin{aligned}
\frac{c}{v_{A 0}}= & \frac{\alpha s \pi}{k L(\exp (\alpha)-1)} \\
& \times\left[1+\frac{\left(4 k^{2} L^{2}-\alpha^{2}\right)(\exp (\alpha)-1)^{2}}{8 \alpha^{2} \exp (\alpha) s^{2} \pi^{2}}+\cdots\right] .
\end{aligned}
$$

This expression is valid for the $s$ th odd harmonic (with $s=1$ corresponding to the first harmonic). It is found that there is excellent agreement for all harmonics, for values of $k L$ less than two.

We can derive a similar approximation for the sth even harmonic multiplying Eq. (22) by -1 and using the expansion for the zeros of

$J_{v}^{\prime}(a) Y_{v}(\lambda a)-J_{v}(\lambda a) Y_{v}^{\prime}(a)$ see Eq. (9.5.33) (p. 374) of Abramowitz \& Stegun (1964). We find

$$
\begin{aligned}
& \frac{c}{v_{A 0}}=\frac{\alpha\left(s-\frac{1}{2}\right) \pi}{k L(\exp (\alpha)-1)} \\
& {\left[1+\frac{\left[k^{2} L^{2}(4 \exp (\alpha)-1)+\alpha^{2}\left(\exp (\alpha)+\alpha^{2}\right)\right](\exp (\alpha)-1)}{8 \alpha^{2} \exp (\alpha)\left(s-\frac{1}{2}\right)^{2} \pi^{2}}+\cdots\right],}
\end{aligned}
$$

with $s=1,2, \ldots$ It should be noted that this expression is not valid for $s=0$, so the fundamental mode cannot be approximated with this formula. Therefore, Eq. (29) provides a good approximation to all harmonics, except the fundamental mode, for values of $c / v_{A 0}$ greater than unity.

\subsubsection{Short wavelength limit}

In the limit of large $k L$ both the order $v$ and argument of the Bessel functions in the dispersion relations (Eqs. (21) and (22)) become large. Therefore, these dispersion relations can be replaced by their leading order expansion from p. 366 of Abramowitz \& Stegun (1964), giving

$v\left[\sqrt{\frac{c^{2} \exp (2 \alpha)}{v_{A 0}^{2}}-1}-\sec ^{-1}\left(\frac{c \exp (\alpha)}{v_{A 0}}\right)\right]=\frac{(4 n-1) \pi}{4}$,

with $n=1,2, \ldots$ for both even and odd modes. Further details for obtaining Eq. (30) are given in the Appendix. The fact that the even and odd modes both have the same asymptotic expansion in this limit is to be expected since their dispersion curves are seen to rapidly converge for increasing $k L$. Since $k L / \alpha$ is becoming large, the term in parenthesis must become small since the right-handside is finite; this happens as $c \exp (\alpha) / v_{A 0}$ approaches 1 . Therefore, using a series expansion for the term in parenthesis about $c \exp (\alpha) / v_{A 0}=1$, we find

$v\left[\frac{2}{3} \sqrt{2}\left(\frac{c \exp (\alpha)}{v_{A 0}}-1\right)^{\frac{3}{2}}\right]=\frac{(4 n-1) \pi}{4}$,

with $n=1,2,3 \ldots$ and therefore

$\frac{c}{v_{A 0}}=\exp (-\alpha)\left(1+\left[\frac{3(4 n-1) \pi}{8 \sqrt{2}} \frac{\alpha}{k L}\right]^{2 / 3}\right)$.

From Eq. (32) it is apparent that the phase speed tends to a lower cutoff value $c \rightarrow v_{A 0} \exp (-\alpha)$ as $k L \rightarrow \infty$, which is in agreement with Fig. 4 and our discussion of the asymptotic values of $c / v_{A 0}$ as $k L \rightarrow \infty$ in Sect. 3.2.2. Note that this approximation also produces the correct limiting behaviour of $c \rightarrow v_{A 0}$ as $\alpha \rightarrow 0$. Good agreement is found between the solution of Eqs. (21) and (22) and the approximate solution in Eq. (32) for the fundamental and first harmonic. As a result of the accuracy of the approximation, being dependent on $(4 n-1) \pi / 4$ for higher harmonics, a much larger value of $k L$ is necessary to observe the same level of agreement as for the fundamental and its first harmonic.

\subsection{Exponential profile with uniform region}

Finally, we consider an Alfvén profile having a similar form to that of the time-averaged profile produced numerically by Mendoza-Briceño et al. (2004), who investigated the heating of a coronal loop, modelled by a 1D hydrodynamic semicircular 


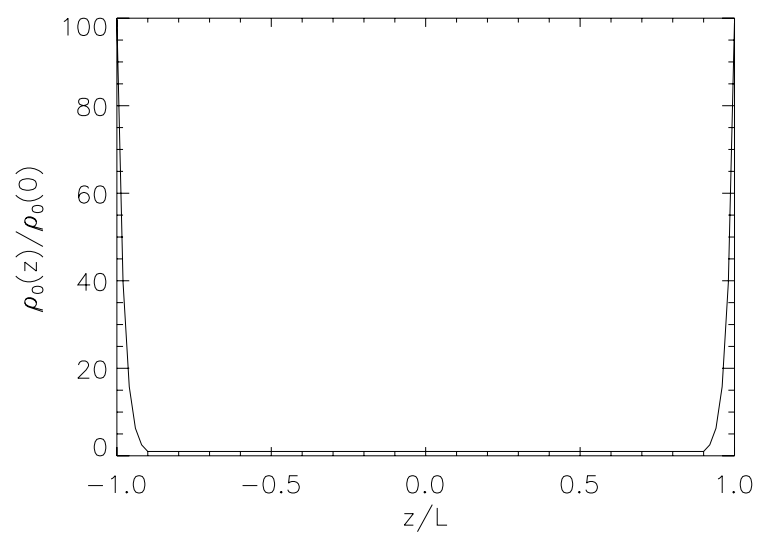

Fig. 7. Dimensionless density profile $\rho_{0}(z) / \rho_{0}(0)$ as a function $z / L$ for a uniform coronal region $|z|<W$ (where is $\rho_{0}(z)=\rho_{0}(0)$ ) and an exponentially stratified chromosphere $W<|z|<L$. The density contrast between footpoints and apex is $\rho_{0}(L) / \rho_{0}(0)=100$ and the coronal depth is $L-W=L / 10$.

simulation. This structure is then subjected to localised impulsive heating near the loop footpoints. This results in a continuously varying temperature profile; when time-averaged, a temperature profile (which corresponds to a density profile) with properties similar to that shown in Fig. 7 is obtained. Unlike the previous case, the density profile in Fig. 7 has the exponential behaviour confined to the footpoints of the structure, where the density changes rapidly from photospheric values to coronal values over the height of the chromosphere, while the central coronal region (from $z=-W$ to $z=W$ ) is uniform. Figure 7 shows the normalised density profile when the chromospheric depth is $(L-W)=L / 10$, one twentieth of the loop length; we have chosen a density contrast of $\rho_{0}(L) / \rho_{0}(0)=100$ between the footpoints and the coronal region.

This density structuring leads to an Alfvén profile of the form

$v_{A}(z)=\left\{\begin{array}{lc}v_{A 0} \exp (\delta(z+W) / L), & -L \leq z \leq-W \\ v_{A 0}, & -W<z<W \\ v_{A 0} \exp (-\delta(z-W) / L), & W \leq z \leq L .\end{array}\right.$

A value of $\delta=23.0$ results in a change to the Alfvén speed between the footpoints and the loop apex by a factor of 10 .

\subsubsection{Dispersion relations}

To derive a dispersion relation, we first note that in the chromospheric region $W<z<L$, Eq. (4) takes the form

$\frac{\mathrm{d}^{2} h}{\mathrm{~d} z^{2}}+\left(\frac{\omega^{2}}{v_{A 0}^{2}} \exp (2 \delta(z-W) / L)-k^{2}\right) h=0$,

with solution

$$
\begin{aligned}
h(z)=A_{2} & J_{\eta}\left(\frac{\omega L}{\delta v_{A 0}} \exp (\delta(z-W) / L)\right) \\
+ & B_{2} Y_{\eta}\left(\frac{\omega L}{\delta v_{A 0}} \exp (\delta(z-W) / L)\right),
\end{aligned}
$$

with $A_{2}$ and $B_{2}$ being arbitrary constants. Equation (35) possesses similar properties as Eq. (17), with non-integer order $\eta=k L / \delta$ and no singularity in the region $[W, L]$, so both Bessel functions are retained. However, the arguments of the Bessel functions are now exponential functions shifted by the coronal depth $W$. In the homogeneous coronal region, $-W<z<W$, Eq. (4) takes the form

$\frac{\mathrm{d}^{2} h}{\mathrm{~d} z^{2}}+M_{0}^{2} h=0$ similar to the homogeneous case (Eq. (7)). However, the boundary conditions we apply at $z= \pm L$ in the homogeneous case are not equivalent to the boundary conditions in Eq. (6) to be applied at $z= \pm W$. As a result $M_{0}^{2}$ is no longer restricted to be positive; hence both oscillatory and hyperbolic functions are admissible under these conditions. Also, as a result of this, the normalised phase speed may fall below $c / v_{A 0}=1$, the lowest value for the entirely uniform case. The general solution $h(z)$ has the form

$h(z)=\left\{\begin{array}{c}C_{1}^{+} \sin \left(M_{0} z\right)+D_{1}^{+} \cos \left(M_{0} z\right), \\ M_{0}^{2} \geq 0 \\ C_{2}^{+} \sinh \left(N_{0} z\right)+D_{2}^{+} \cosh \left(N_{0} z\right), \\ M_{0}^{2}=-N_{0}^{2}<0,\end{array}\right.$

with $C_{1}^{+}, C_{2}^{+}, D_{1}^{+}$and $D_{2}^{+}$, being arbitrary constants.

Application of the appropriate boundary conditions in Eq. (19) and (20) at $z=0$ allows us to separate the discussion into even and odd modes. In this case there is also an interface at $z= \pm W$, where the continuity of both $h(z)$ and $\frac{d h}{d z}$ must be enforced. This leads us to the following transcendental dispersion relations (for the case $M_{0}^{2}>0$ ):

$\frac{c}{v_{A 0}} \tan \left(k W \sqrt{\frac{c^{2}}{v_{A 0}^{2}}-1}\right)=\frac{F(c, k) \sqrt{\frac{c^{2}}{v_{A 0}^{2}}-1}}{G(c, k)}$

for the odd modes, and

$-\frac{c}{v_{A 0}} \cot \left(k W \sqrt{\frac{c^{2}}{v_{A 0}^{2}}-1}\right)=\frac{F(c, k) \sqrt{\frac{c^{2}}{v_{A 0}^{2}}-1}}{G(c, k)}$

for the even modes. Here we have written

$$
\begin{aligned}
F(c, k)=J_{\eta} & \left(\frac{c k L}{\delta v_{A 0}} \exp \left(\delta \frac{L-W}{L}\right)\right) Y_{\eta}\left(\frac{c k L}{\delta v_{A 0}}\right) \\
& -Y_{\eta}\left(\frac{c k L}{\delta v_{A 0}} \exp \left(\delta \frac{L-W}{L}\right)\right) J_{\eta}\left(\frac{c k L}{\delta v_{A 0}}\right)
\end{aligned}
$$

and

$$
\begin{aligned}
G(c, k)=J_{\eta} & \left(\frac{c k L}{\delta v_{A 0}} \exp \left(\delta \frac{L-W}{L}\right)\right) Y_{\eta}^{\prime}\left(\frac{c k L}{\delta v_{A 0}}\right) \\
& -Y_{\eta}\left(\frac{c k L}{\delta v_{A 0}} \exp \left(\delta \frac{L-W}{L}\right)\right) J_{\eta}^{\prime}\left(\frac{c k L}{\delta v_{A 0}}\right) .
\end{aligned}
$$

In these expressions we have assumed $M_{0}^{2}>0$; equivalent relations arise in the case $M_{0}^{2}<0$ with hyperbolic functions replacing trigonometric functions. Notice there is no upper cutoff frequency but we find that there is a lower cutoff value, which the dispersion curves approach in the short wavelength limit. This cutoff lies in the range $[\exp (\delta(W / L-1)), 1]$; the same argument as the previous case can be made once $c / v_{A 0}<1$, which is equivalent to $M_{0}^{2}<0$. The cutoff value returns to the case of a uniform medium, when $\delta \rightarrow 0$ or $W \rightarrow L$.

\subsubsection{Dependence on the wavenumber}

Figure 8a shows the dispersion diagram plotting the dimensionless phase speed $c / v_{A 0}$ against dimensionless wavenumber $k L$. We have taken $\delta=23$, which results in a density contrast of 100 between the loop apex and footpoint. As in the previous case, no mode propagates with finite phase speed in the limit $k L \rightarrow 0$ and there is a lower cutoff value to which the modes tend in the limit $k L \rightarrow \infty$. However, comparing with Fig. 2a, the spacing of the dispersion curves has been modified and this influences the period ratio $P_{1} / 2 P_{2}$ shown in Fig. 9.

The phase speed of the odd and even modes virtually coincide on crossing the curve $c / v_{A 0}=1$ in Fig. 8a (and in Fig. 8b 


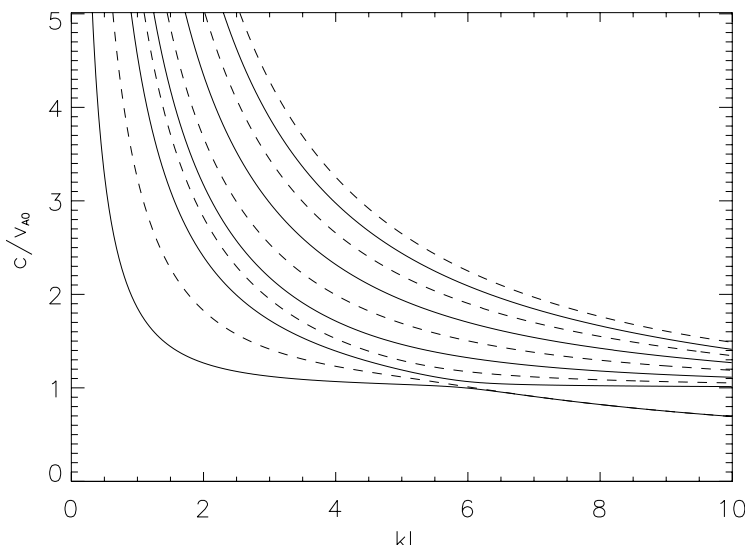

(a)

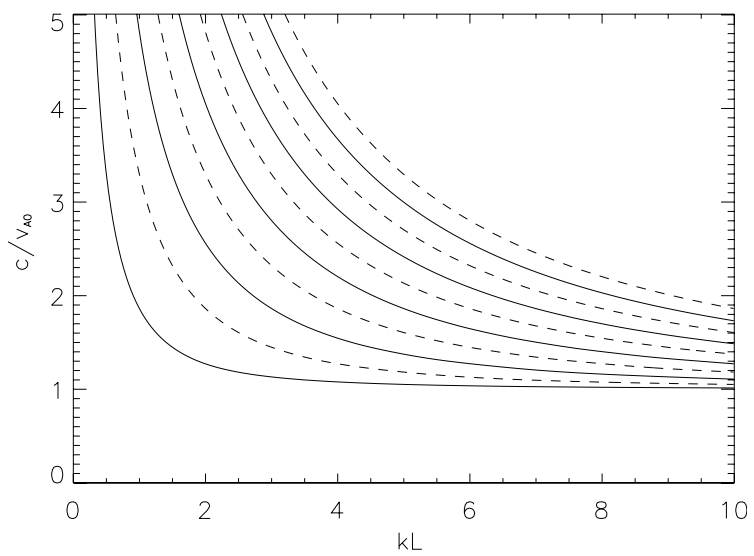

(b)

Fig. 8. Dispersion diagrams plotting dimensionless phase speed $c / v_{A 0}$ against $k L$ for the Alfvén profile in Eq. (33) with $L-W=L / 10$ and density contrasts a) $\rho_{0}(L) / \rho_{0}(0)=100(\delta=23.0)$ and b) $\rho_{0}(L) / \rho_{0}(0)=1.2$ $(\delta=0.1)$. The even and odd modes are plotted as solid and dashed curves, respectively. Only the fundamental mode and its first nine harmonics are shown

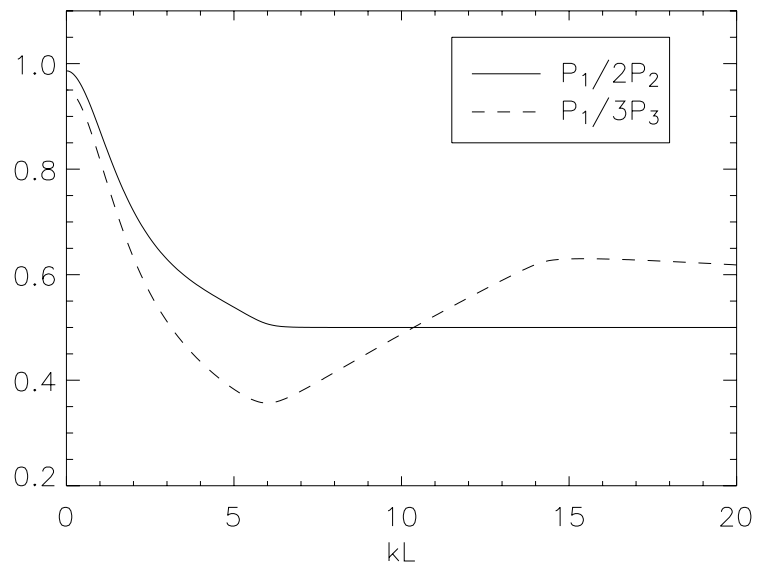

Fig. 9. The ratios $P_{1} / 2 P_{2}$ (solid line) and $P_{1} / 3 P_{3}$ (dashed line) plotted against $k L$ for and exponentially stratified arcade with homogeneous region at the apex, with $\rho_{0}(L) / \rho_{0}(0)=100$ and $L-W=L / 10$.

for larger values of $k L$, although not shown in the plot). This is because the trigonometric functions in Eqs. (38) and (39) become hyperbolic functions for $c / v_{A 0}<1$ and tanh $a \approx \operatorname{coth} a$ for large $a$; since this condition is satisfied only when $k L$ is large, the dispersion relations are very similar in that limit. For $c / v_{A 0}>1$, the phase speed of the even and odd modes are distinct. There

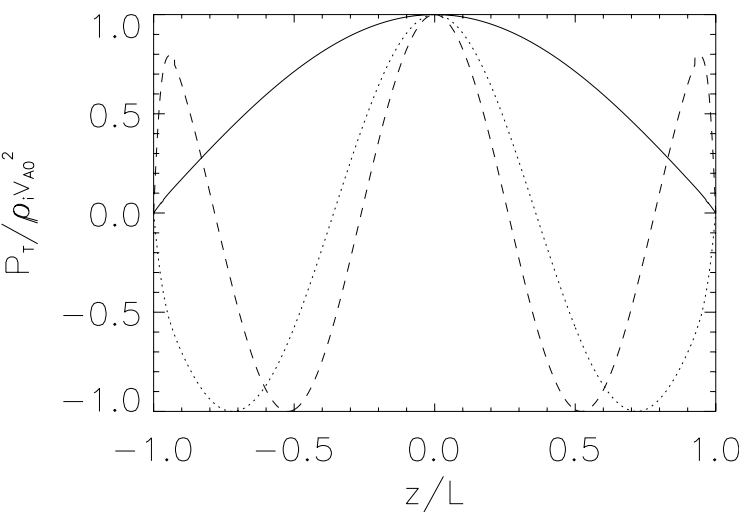

(a)

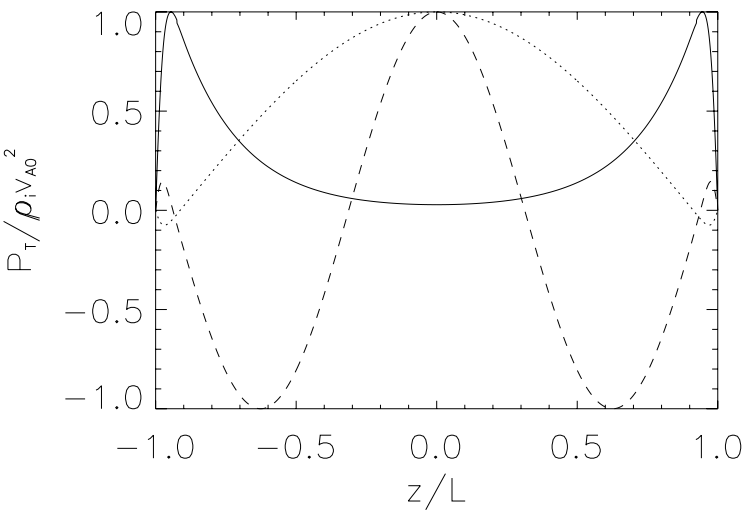

(b)

Fig. 10. Plots of the dimensionless total pressure perturbation $P_{T} /\left(\rho_{0} v_{A 0}^{2}\right)$ against $z / L$ for the fundamental (solid curve), first (dotted curve) and second (dashed curve) even harmonics, for a) $k L=2$ and b) $k L=8$. The model has chromospheric layers of depth $L-W=L / 10$ with a density enhanced by a factor of 100 between the loop apex and the footpoints.

is an avoided crossing between the first two even modes at the point where the curve corresponding to the fundamental mode crosses $c / v_{A 0}=1$. This is because on crossing this curve the eigenfunctions can have at most one extrema in the central region of constant equilibrium density $0<z<W$ (which is located at the summit), due to their hyperbolic dependence; hence if more extrema are present before crossing this curve they must be transferred to the region with an exponentially increasing equilibrium density, $W<z<L$ (located at the footpoint), via an avoided crossing. As a result of this avoided crossing, local minima and maxima can be found in the ratio $P_{1} / 3 P_{3}$ (see Fig. 9) so it is no longer a monotonic decreasing function of $k L$, as arose in the homogeneous case (Fig. 2b) or the exponential case (Fig. 5). However, setting a low value for the density contrast between footpoints and apex, such as $\delta=0.1$, we find that we recreate the uniform medium case (as seen in Fig. 8b, where the curves tend to $c / v_{A 0}=1$ for larger values of $k L$ than those shown in the plot).

Figure 10 shows plots of the dimensionless total pressure perturbation (normalised to 1 at its maximum value) as a function of $z / L$, for the fundamental $(n=1)$, first $(n=3)$ and second $(n=5)$ even harmonics for values of $k L=2$ (Fig. 10a) and $k L=8$ (Fig. 10b). For all of the modes plotted at $k L=2$, we find that $c / v_{A 0}>1$ and so $M_{0}^{2}>0$ (see Fig. 8a); hence the total pressure perturbation is oscillatory in the central region $|z|<W$. The fundamental mode takes a similar form to that of a uniform arcade (the cosine function in Eq. (8)), with one extrema for a 


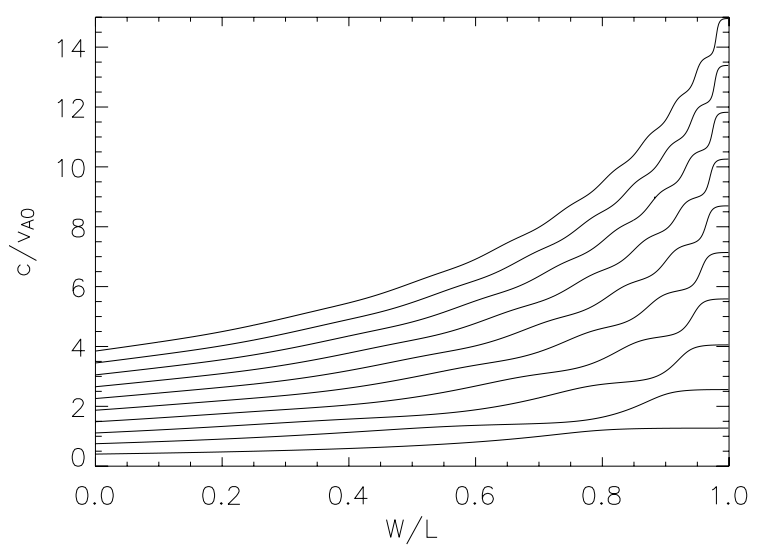

(a)

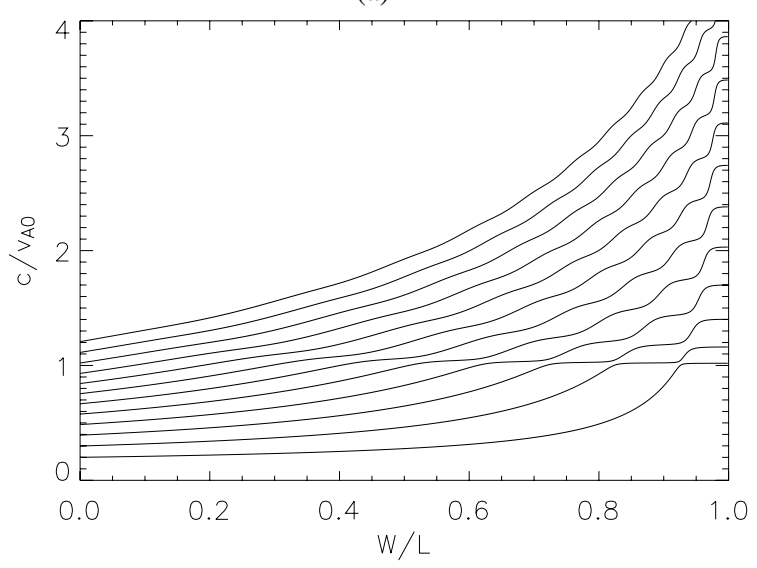

(b)

Fig. 11. Dispersion diagrams plotting $c / v_{A 0}$ against $W / L$ (even modes) for a density profile with chromospheric layers and a density contrast $\rho_{0}(L) / \rho_{0}(0)=100$, with a) $k L=2$ and b) $k L=8$.

value of $k L=2$ (Fig. 10a, solid curve). But comparing this to the fundamental mode for $k L=8$ (Fig. 10a, solid curve), after the avoided crossing between this fundamental mode and first harmonic, we see it now has three extrema while the first harmonic at $k L=8$ (Fig. 10b, dotted curve) has a very similar behaviour to the fundamental mode at $k L=2$ (Fig. 10a, solid curve), since they are connected through an avoided crossing. In the regions $W<|z|<L$, the solutions have smaller amplitudes but a greater number of extrema in a short distance for both $k L=2$ and $k L=8$. These oscillations with large wavenumber in the direction along the magnetic field ( $z$-direction) are similar to the findings of Díaz \& Roberts (2006) for the behaviour of slow magnetoacoustic modes.

\subsubsection{Dependence on the chromospheric depth}

It is of interest to investigate the influence of the chromospheric depth $(L-W)$ on the phase speed of these modes. Plots of phase speed against $W / L$ are shown in Fig. 11 for a density ratio of $\rho_{0}(L) / \rho_{0}(0)=100$. To achieve this density ratio for various $W / L$ we vary $\delta$, these quantities being related by

$\delta=\frac{1}{2\left(1-\frac{W}{L}\right)} \ln \frac{\rho_{0}(L)}{\rho_{0}(0)}$.

The plots provide us the opportunity to compare the phase speeds of the uniform medium $(W / L=1)$ and the purely

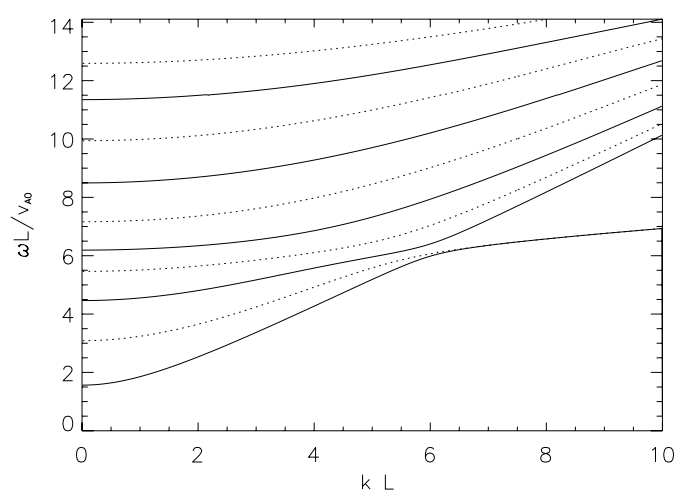

Fig. 12. Dispersion diagram plotting $\omega L / v_{A 0}$ against $k L$ for the Alfvén profile in Eq. (33) with $L-W=L / 10$ and density contrast $\rho_{0}(L) / \rho_{0}(0)=$ 100 . The even and odd modes are plotted as solid and dashed curves, respectively.

exponentially structured case $(W / L=0)$. We see that stratification results in a reduction of phase speed.

Figure 11a shows a plot of $c / v_{A 0}$ for $k L=2$. Immediately we notice a large number of interactions between adjacent modes, as a result of the many small amplitude oscillations in the chromospheric region being transferred from the coronal region. The phase speed $c$ falls from its values in the homogeneous structure $(W / L=1)$ to an entirely exponential density profile $(W / L=0)$, since more dense material is being considered as $W / L$ is increased. The fundamental mode shows little dependence on the chromospheric depth. We can still see many of these features if we increase the wavenumber (for example, $k L=8$ in Fig. 11b), though now the fundamental mode is strongly dependent on the chromospheric depth in the range $W / L=0.3-0.9$; however, this feature happens outside the realistic thin chromosphere region, in which the phase speed does not depend on $W / L$.

\section{Comparison with observations}

Observations of a flare excited oscillation in a coronal arcade have recently been discussed in Verwichte et al. (2004). Finding a coherent oscillation of many coronal loops forming an arcade structure, there is strong evidence in oscillation period and also in displacement profile (in the plane of the loop) that not only the fundamental but also the first harmonic are being observed. We may test the model presented in Sect. 3.3 against the observational data in Verwichte et al. (2004) by using the period $P_{1}$ of the fundamental mode to fix the parameters of the model, and then deducing the period of the first harmonic $P_{2}$ to compare it with to the observed value.

The height of the loop arcade is in the range 65-76 Mm (Verwichte et al. 2004). We choose a value of $70 \mathrm{Mm}$ and assume the loop arcade is semicircular, leading to a field line length of $70 \pi \mathrm{Mm}=220 \mathrm{Mm}$. We assume that the density profile along the loop is the same as presented in Sect. 3.3, where each chromospheric layer has been taken to be one twentieth of the total loop length and a density contrast of 100 occurs between the chromosphere and the coronal structure; we take a coronal Alfvén speed of $1000 \mathrm{~km} \mathrm{~s}^{-1}$ (assumed uniform).

Taking the observed period to be $400 \mathrm{~s}$ (Verwichte et al. $2004)$, it corresponds to a dimensionless frequency of $\omega L / v_{A 0}=$ 1.72 for the fundamental mode. Reading the wavenumber from Fig. 12, we find that the mode is propagating with a wavenumber of $k L=0.72$. Assuming that the first harmonic (i.e., the lowest odd mode) has a similar wavenumber (not unexpected 
because $k L$ is the wavenumber in the direction the wave packet is propagating and so is independent of the longitudinal direction), this allows us to read the frequency of the first harmonic from Fig. 12: $\omega L / v_{A 0}=3.16$, giving a period of $218 \mathrm{~s}$ for the first harmonic. This is in good agreement with the observed value of $242 \pm 31$ s (Verwichte et al. 2004).

However, it must be remarked that we have used the value of the period of the fundamental mode to obtain the wavenumber, and then checked that the period of the first harmonic is in accordance with the observational data. One could carry this further and use the observed period ratio $P_{1} / 2 P_{2}$ to obtain a wavenumber in Fig. $9(k L=1.27$ with the values in Verwichte et al. 2004), and then use it in Fig. 12 to obtain a value of for the dimensionless frequency of the fundamental mode $\left(\omega L / v_{A 0}=2,02\right)$, and from here a value for the coronal Alfvén speed $\left(v_{A 0}=853 \mathrm{~km} \mathrm{~s}^{-1}\right)$, which is in the expected range for the solar corona. Hence, we obtain an equilibrium parameter via coronal seismology. However, taking into account the uncertainties in the determination of the periods, the values obtained here just point out that the agreement between our estimate and the expected values for the equilibrium parameters and arcade properties is acceptable. Notice also that a different value of $W$ or $\delta$ would have given different values for the transversal wavenumber $k L$ and the coronal Alfvén speed, so these parameters must be also inferred from the observations.

\section{Conclusions}

Our aim here has been to model the oscillations of a coronal arcade with footpoints fixed in the photospheric surface, allowing for free propagation along the arcade (across the magnetic field lines). We considered a uniform magnetic field directed in the $z$-direction, along with a density $\rho_{0}(z)$ varing with distance. We solved the linearised MHD equations in the zero beta limit, so only the fast modes are discussed.

A homogeneous arcade provides a reference case, which allows us to derive an explicit dispersion relation (Eq. (12)). From this dispersion relation we find that the phase speed $c$ tends to the limit $c=v_{A 0}$ (see Fig. 2a). Andries et al. (2005a) identified the ratio $P_{1} / 2 P_{2}$ of periods of the fundamental mode $P_{1}$ to its first harmonic $P_{2}$ to be potentially important in the field of coronal seismology, since it provides an observational quantity that can be related with the equilibrium density structure, while the fundamental mode frequency depends only slightly on it (see also Díaz et al. 2006a; McEwan et al. 2006; Dymova \& Ruderman 2006; Erdélyi \& Verth 2007). In the homogeneous case the ratio $P_{1} / 2 P_{2}$ is unity in the long wavelength limit and monotonically decreases to $1 / 2$ in the short wavelength limit (see Fig. 2b).

The second case examined here is that of an exponential density profile, with the loop footpoints denser than the apex. We have taken the density enhancement to be a factor of 100 . The addition of the density structure results in transcendental dispersion relations, Eqs. (21) and (22), for odd and even modes, respectively. From this, we find a reduction in the phase speed compared with the homogeneous case. The phase speed of consecutive even and odd modes converge for large $k L$, which has been demonstrated analytically. As in the homogeneous case, there is a lower cutoff value for the phase speed, which lies not at $c=v_{A 0}=1$, but at $c / v_{A 0} \approx \exp (-\alpha)$. The fact that the dispersion curves are differently spaced influences the period ratio $P_{1} / 2 P_{2}$, causing it to be less than unity in the limit $k L \rightarrow 0$; but as $k L \rightarrow \infty$ the period ratio $P_{1} / 2 P_{2}$ still tends to $1 / 2$. In this case the eigenfunctions exhibit a behaviour different to the homogeneous case, developing many extrema close together in the footpoint regions. Also, in some cases the fundamental mode has more than one extrema. We are able to recover the properties of the homogeneous case in the limit $\alpha \rightarrow 0$.

The drawback of the density profile we discuss is that in reality the change from photospheric to coronal densities occurs more rapidly. However, the third profile we considered does not have this drawback, since it has exponential behaviour in the chromospheric region $W<|z|<L$ and is uniform in the corona $|z|<W$. Importantly, testing this model as $\delta \rightarrow 0$ or $W \rightarrow L$ shows that it reduces to the homogeneous case, and also as $W \rightarrow 0$ to the exponential profile case. We can see in Fig. 11 that the decrease in phase speed for the fundamental period induced by the longitudinal structuring is relatively small compared with the homogeneous case, so the results of the homogeneous arcade provide a good approximation for $P_{1}$; this is a akin to the results of structured loops (Andries et al. 2005b; Díaz et al. 2006a). Crossing the curve $c / v_{A 0}=1$, the phase speeds of consecutive even and odd modes rapidly converge. Also, on crossing this curve we found an interaction between the fundamental and first harmonic of the even modes in the form of an avoided crossing. As expected, the floor value is altered by the structure and now lies in the range $[\exp (-\delta(W / L-1)), 1]$. The influence of this case on the ratio $P_{1} / 2 P_{2}$ is the same as the previous case.

It is also interesting to consider $P_{1} / 3 P_{3}$, which is less than 1 as $k L \rightarrow 0$. This case also has a local minimum, as a result of avoided crossings between these two modes; this type of behaviour could also arise in $P_{1} / 2 P_{2}$ in a model where these modes did not decouple (such as a non-symmetric equilibrium). The eigenfunctions display oscillatory behaviour in the region $|z|<W$ and more rapid oscillations in $W<|z|<L$, and the exchange of extrema between these two regions can account for the large number of avoided crossings seen in Fig. 11 as $W / L$ varies. This model has been shown to compare well with observations of an impulsively excited arcade oscillation; when given the period of the fundamental mode, the period of the first harmonic has been calculated and is found to be consistent with observed values. Moreover, using the approach of coronal seismology, we have estimated the values of the longitudinal wavenumber and coronal Alfvén speed which can be deduced from these observations and (despite the hypothesis and simplifications carried out in our model) the values we obtain are in the expected range for coronal arcades.

Curvature effects have been ignored in this paper, where we have focused on the effect of structure. Curvature has not a major impact on the frequencies, but introduces other important modifications (Verwichte et al. 2006a,b; Díaz et al. 2006b), such as leakage and the presence of new families of modes. However, the introduction of transversal propagation in curved arcades couples the governing equations, so it is no longer possible to study the Alfvén and the fast modes separately, and the longitudinal structure adds further complexity. However, the results found in the present paper provide a guidance for the type of modifications that these two effects may introduce, but further work is needed to have models more appropriate for a full description of the oscillations of coronal arcades.

It is clear that the presence of structure, regardless of the effect producing it, has an important influence on both the frequency of modes and their eigenfunctions. Due to the shift in frequency caused by the structure, the seismological tool $P_{1} / 2 P_{2}$ is also modified and the allowance of cross-field propagation $(k \neq 0)$ further complicates this effect. This may be important in future developments in coronal seismology.

Acknowledgements. G.R.D. acknowledges financial support from the Particle Physics and Astronomy Research Council. A.J.D. acknowledges financial 
support from PPARC on the St Andrews Solar Theory Rolling Grant. The authors also thank the referee for useful comments and suggestions

\section{References}

Abramowitz, M., \& Stegun, I. A. 1964, Handbook of Mathematical Functions (National Bureau of Standards)

Andries, J., Arregui, I., \& Goossens, M. 2005a, ApJ, 624, L57

Andries, J., Goossens, M., Hollweg, J. V., Arregui, I., \& Van Doorsselaere, T. 2005b, A\&A, 430, 1109

Aschwanden, M. J. 2004, Physics of the Solar Corona (Berlin: Springer)

Aschwanden, M. J., De Pontieu, B., Schrijver, C. J., \& Title, A. M. 2002, Sol. Phys., 206, 99

Brady, C. S., \& Arber, T. D. 2005, A\&A, 438, 733

Díaz, A. J. 2004, Ph.D. Thesis, Departament de Física, Universitat de les Illes Balears, Palma Mallorca

Díaz, A. J. 2006, A\&A, 456, 737

Díaz, A. J., \& Roberts, B. 2006, A\&A, 458, 975

Díaz, A. J., Oliver, R., \& Ballester, J. L. 2002, ApJ, 580, 550

Díaz, A. J., Oliver, R., Ballester, J. L., \& Roberts, B. 2004, A\&A, 424, 1055

Díaz, A. J., Oliver, R., \& Ballester, J. L. 2006a, ApJ, 645, 766

Díaz, A. J., Zaqarashvili, T., \& Roberts, B. 2006b, A\&A, 455, 709
Dymova, M. V., \& Ruderman, M. S. 2006, A\&A, 457, 1059

Edwin, P. M., \& Roberts, B. 1983, Sol. Phys., 88, 179

Erdélyi, R., \& Verth, G. 2007, A\&A, 462, 743

Goedbloed, J. P. 1983, Lecture Notes on Ideal Magnetohydrodynamics (Rijnhuizen Report), 76

Goossens, M., Andries, J., \& Arregui, I. 2006, Phil. Trans. R. Soc., 364, 433

Hood, A. W. 1986, Sol. Phys., 105, 307

McEwan, M. P., Donnelly, G. R., Díaz, A. J., \& Roberts, B. 2006, A\&A, 460, 893

Mendoza-Briceño, C. A., Erdélyi, R., \& Sigalotti, L. D. G. 2004, ApJ, 605, 493 Nakariakov, V. M., \& Ofman, L. 2001, A\&A, 372, L53

Nakariakov, V. M., \& Verwichte, E. 2005, Liv. Rev. Sol. Phys., 2, 3

Oliver, R., Ballester, J. L., Hood, A. W., \& Priest, E. R. 1993, A\&A, 273, 647

Roberts, B. 1991, in Advances in Solar System Magnetohydrodynamics, ed.

E. R. Priest, \& A. W. Hood (Cambridge University Press), 105

Roberts, B., Edwin, P. M., \& Benz, A. O. 1984, ApJ, 279, 857

Selwa, M., Murawski, K., Solanki, S. K., Wang, T. J., \& Tóth, G. 2005, A\&A, 440, 385

Smith, J. M., Roberts, B., \& Oliver, R. 1997, A\&A, 317, 752

Verwichte, E., Nakariakov, V. M., Ofman, L., \& Deluca, E. E. 2004, Sol. Phys., 223, 77

Verwichte, E., Foullon, C., \& Nakariakov, V. M. 2006a, A\&A, 446, 1139

Verwichte, E., Foullon, C., \& Nakariakov, V. M. 2006b, A\&A, 449, 769 
G. R. Donnelly et al.: Effect of longitudinal structure on coronal arcade oscillations, Online Material p 1

\section{Online Material}


G. R. Donnelly et al.: Effect of longitudinal structure on coronal arcade oscillations, Online Material p 2

\section{Appendix A: Deduction of the dispersion relation \\ on the short wavelength limit}

In the investigation of dispersion relation Eq. (21) for the modes of an exponential equilibrium density profile, namely

$$
J_{v}\left(v \frac{c \exp (\alpha)}{v_{A 0}}\right) Y_{v}\left(v \frac{c}{v_{A 0}}\right)-J_{v}\left(v \frac{c}{v_{A 0}}\right) Y_{v}\left(v \frac{c \exp (\alpha)}{v_{A 0}}\right)=0,
$$

it is of interest to examine the behaviour for large $k L$. This requires the use of expansions of Bessel functions $J_{v}(\lambda v)$ and $Y_{v}(\lambda v)$ as $v \rightarrow \infty$. The cases $\lambda>1$ and $0<\lambda<1$ are different.

The solution of Eq. (16) must have a region inside $[0, L]$ where it is oscillatory in nature, and somewhere in this region

$\frac{c^{2} \exp (2 \alpha z / L)}{v_{A 0}^{2}}-1>0$

is satisfied. So, if there is a solution, then this condition must be satisfied at $z=L$, and then

$\frac{c \exp (\alpha)}{v_{A 0}}>1$.

Therefore the expansions

$$
\begin{aligned}
& J_{v}\left(v \frac{c \exp (\alpha)}{v_{A 0}}\right) \sim \sqrt{\frac{2}{\pi v \sqrt{\frac{c^{2} \exp (2 \alpha)}{v_{A 0}^{2}}-1}}} \cdot \\
& \quad \cos \left(v \sqrt{\frac{c^{2} \exp (2 \alpha)}{v_{A 0}^{2}}-1}-v \sec ^{-1}\left(\frac{c \exp (\alpha)}{v_{A 0}}\right)-\frac{\pi}{4}\right)
\end{aligned}
$$

and

$$
\begin{aligned}
& Y_{v}\left(v \frac{c \exp (\alpha)}{v_{A 0}}\right) \sim \sqrt{\frac{2}{\pi v \sqrt{\frac{c^{2} \exp (2 \alpha)}{v_{A 0}^{2}}-1}}} \cdot \\
& \quad \sin \left(v \sqrt{\frac{c^{2} \exp (2 \alpha)}{v_{A 0}^{2}}-1}-v \sec ^{-1}\left(\frac{c \exp (\alpha)}{v_{A 0}}\right)-\frac{\pi}{4}\right)
\end{aligned}
$$

are employed. On the other hand, from the solution of the full dispersion relation we obtain $c / v_{A 0}<1$ as $k L \rightarrow \infty$, so we use for the remaining Bessel functions in Eq. (A.1) the expansions:

$J_{v}\left(v \frac{c}{v_{A 0}}\right) \sim \frac{\exp \left[v\left(\sqrt{1-\frac{c^{2}}{v_{A 0}^{2}}}-\operatorname{sech}^{-1}\left(\frac{c}{v_{A 0}}\right)\right)\right]}{\sqrt{2 \pi v \sqrt{1-\frac{c^{2}}{v_{A 0}^{2}}}}}$ and

$Y_{v}\left(v \frac{c}{v_{A 0}}\right) \sim-\frac{\exp \left[-v\left(\sqrt{1-\frac{c^{2}}{v_{A 0}^{2}}}-\operatorname{sech}^{-1}\left(\frac{c}{v_{A 0}}\right)\right)\right]}{\sqrt{\frac{1}{2} \pi v \sqrt{1-\frac{c^{2}}{v_{A 0}^{2}}}}}$.

Now substituting expansions (A.4), (A.5), (A.6) and (A.7) in dispersion relation (A.1), we obtain the expression for the dispersion relation for large $k L$. However, since

$\sqrt{1-\frac{c^{2}}{v_{A 0}^{2}}}-\operatorname{sech}^{-1}\left(\frac{c}{v_{A 0}}\right) \leq 0$

the expansion in Eq. (A.6) goes to zero, and hence the second term in Eq. (A.1) goes to zero. Therefore, Eq. (A.1) is satisfied when

$$
\begin{aligned}
\cos \left(v \sqrt{\frac{c^{2} \exp (2 \alpha)}{v_{A 0}^{2}}-1}\right. \\
\left.\quad-v \sec ^{-1}\left(\frac{c \exp (\alpha)}{v_{A 0}}\right)-\frac{\pi}{4}\right)=0
\end{aligned}
$$

This is equivalent to

$$
\begin{aligned}
v\left(\sqrt{\frac{c^{2} \exp (2 \alpha)}{v_{A 0}^{2}}-1}\right. \\
\left.\quad-\sec ^{-1}\left(\frac{c \exp (\alpha)}{v_{A 0}}\right)\right)=\frac{(4 n-1) \pi}{4},
\end{aligned}
$$

which is Eq. (30).

A similar approach is can be used to derive an identical expression for the asymptotic behaviour of the even modes described in the dispersion relation of Eq. (22). 